Classification

Physics Abstracts

$78.60 \mathrm{H}-68.65-73.40 \mathrm{~L}$

\title{
The study of individual free standing GaAs/AlGaAs quantum-dots by STEM-CL
}

\author{
Jiannong Wang $\left({ }^{1}\right)$, John Wickham Steeds $\left({ }^{1}\right)$ and Hazel Arnot $\left({ }^{2}\right)$ \\ ( ${ }^{1}$ ) H.H. Wills Physics Laboratory, University of Bristol, Bristol BS8 1TL, G.B. \\ $\left({ }^{2}\right)$ Departement of Electronic and Electrical Engineering, University of Glasgow, \\ Glasgow G12 8QQ, G.B.
}

(Received October 18, 1990; accepted November 15, 1990)

\begin{abstract}
Résumé. - Des points quantiques libres, fabriqués par gravure ionique réactive à partir d'une structure de puits quantiques de GaAs/A1GaAs, sont étudiés. La surface directement attaquée, est sévèrement endommagée. On detecte la luminescence d'un point quantique. On observe une réduction de la luminescence provenant de ces points. Finalement, des images de luminescence filtrées en longueur d'onde, ont permis de visualiser des points quantiques individuels organisés en réseau.
\end{abstract}

\begin{abstract}
Individual free-standing quantum-dots fabricated from a GaAs/A1GaAs quantum well structure by reactive-ion etching have been investigated. Severe damage occurred at the directly etched surface. Luminescence from a single quantum dot was detected. A loss of luminescence efficiency from the dots was observed. Energy-selected luminescence images of individual quantum-dots in an array were obtained.
\end{abstract}

\section{Introduction.}

Many novel optical and transport properties have been observed as bulk materials are reduced in thickness to create two-dimensional, quantum-well structures. One expects additional new physical phenomena to emerge if the dimensions of the materials can be reduced further. In the case of zero-dimensional quantum-dots (also called quantum-boxes or quantum disks) the effect of confinement in all three directions is to modify the density of states and increase the interaction between electrons and holes [1]. As a result large electroabsorption, refraction, and nonlinear optical effects are predicted.

Recently, much attention has been focused on the fabrication of quantum-dots from semiconductor quantum-well structures and their characterization by a variety of techniques. Generally speaking, two different methods have been applied to produce quantum-dots from quantumwell (QW) structures. Buried quantum dots can be formed with high resolution electron beam lithography by a combination of ion-implantation-induced interdiffusion in $\mathrm{QW}$ structures. On the other hand, free-standing quantum dots can be fabricated by the application to quantum-well 
structures of electron-beam lithography patterning and dry etching [1-3]. Most research work has been concentrated on the GaAs/A1GaAs system. In the latter method, there is a tendency for sidewall surface damage to occur during the etching process, leading to a high non-radiative surface recombination velocity. In addition, there will be a surface layer depleted of carriers. A degradation of luminescence efficiency has been observed as the lateral sizes of the dots approached the carrier diffusion length $(\sim 1 \mu \mathrm{m})[1,4]$. A depletion layer width of $100-150 \mathrm{~nm}$ was found at the sidewalls by optical measurements [1] and magnetotransport measurements [5]. However, the results of luminescence studies of etched, free standing GaAs/a1GaAs quantum dots are inconsistent. Other studies have claimed no degradation of luminescence efficiency occurred for quantum dot structures with the lateral sizes down to $40 \mathrm{~nm}$ [6-8]. However, all the results of these past studies were carried out on quantum-dot arrays, which contain many individual dots, and reflected the average effects of a entire array. No studies of individual quantum-dots have been reported, even though it is clear that this is an important issue.

In this paper, by applying the technique of low-temperature and high spatial resolution CL (cathodoluminescence) we present the results of a study of individual free-standing quantumdots fabricated from a GaAs/A1GaAs quantum well structure. Images of individual quantum-dots in an array were formed by energy-selected luminescence emission. CL spectra from individual quantum -dots were also obtained. A degradation of the luminescence efficiency of individual quantum-dots occured. A weaker deterioration was also observed on the luminscence efficiency for quantum-dots fabricated from narrower quantum-wells.

\section{Experimental details.}

In these experiments, the GaAs/A1GaAs quantum dots were formed from the quantum well structure shown in figure 1 , which was grown by molecular beam epitaxy on an $n+$ GaAs substrate. The structure contained four single quantum wells with nominal GaAs well-widths of $50 \AA, 100 \AA, 200$ $\AA$ and $800 \AA$ and $\mathrm{A} 1 \mathrm{GaAs}$ barrier thicknesses of $340 \AA$. The free-standing quantum dots were produced by a combination of electron beam lithography patterning and reactive ion-etching using methane/hydrogen $\left(\mathrm{CH}_{4} / \mathrm{H}_{2}\right)$. The etching depth was $800 \AA$, which ensured that the $50 \AA$ and $100 \AA$ wells formed into dots. A large unetched area (50 $\mu \mathrm{m}$ in size) was preserved in the sample as a processing control to evaluate the luminescence efficiency of the layers. An array with dots $500 \mathrm{~nm}$ in diameter and $2 \mu \mathrm{m}$ in spacing has been studied.

Low temperature and high spatial resolution CL experiments were performed in a extensively modified Philips EM400 Scanning Transmission Electron Microscope. A detailed description of the CL system can be found elsewhwere [9-11]. In brief, a retractable and adjustable ellipsoidal mirror, with a small hole to allow the passage of the electron beam, is used to collect the light generated by the incident electron beam. The beam impinges on the specimen at one focus of the mirror. The light is collected by the mirror, brought out of the microscope, and reflected through quartz optics into a $50 \mathrm{~cm}$ path length monochrometer. The detector used in this work was an RCA 31034A photomultiplier with a GaAs photocathode. The specimen was cooled down to about $30 \mathrm{~K}$ by mounting it in a cold stage with continuous flow of liquid helium. A $20 \mathrm{kV}$ electron beam was choosen as excitation source. In STEM mode the dots could be resolved in secondary-electron images. This enabled us to identify individual dots within the arrays so that low temperature CL investigations could be performed on any chosen dot.

\section{Results and discussion.}

Luminescence spectra acquired from the large unetched areas represents the QW emission from the structure prior to etching. An example is shown in figure 2 (curve (a)). The peaks at $1.591 \mathrm{eV}$, 


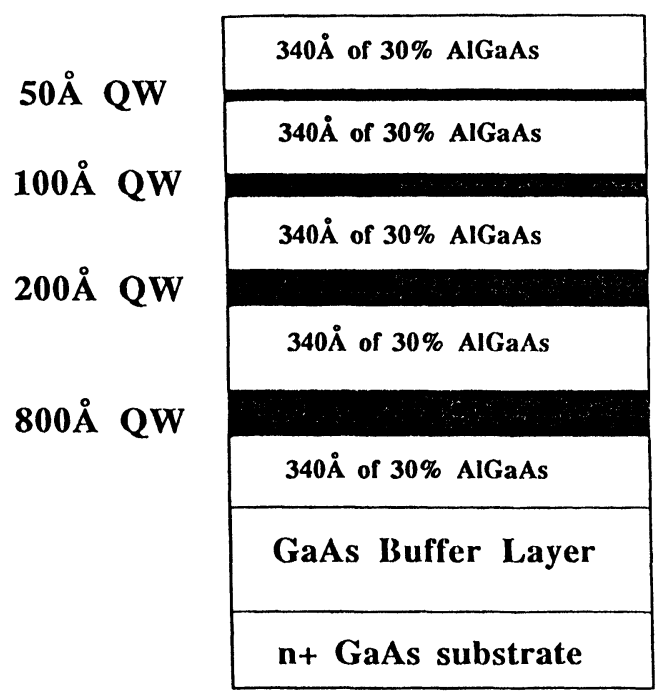

Fig. 1. - Schematic diagram showing the detailed structure of the quantum-well sample from which the quantum-dots were fabricated.

$1.543 \mathrm{eV}$ and $1.521 \mathrm{eV}$ correspond to the $\mathrm{QW}$ excitonic emission from the $50 \AA, 100 \AA$ and $200 \AA$ wells respectively. Emission from $800 \AA$ well overlapped the GaAs bulk emission at $1.514 \mathrm{eV}$ from the buffer layer and substrate. The low energy tail of the $1.514 \mathrm{eV}$ emission may have resulted from impurities in the GaAs buffer or in the substrate. In the areas where the quantum-dots had been fabricated, the luminescence spectra from the etched areas, which still contained the $200 \AA$ and $800 \AA$ quantum-wells, had changed greatly from that shown in figure 2 curve (a). As shown in figure 2 (curve (b)), the low-energy tails in curve (a) from the bulk GaAs layers dominated the whole spectrum. The high energy shoulders at $1.517 \mathrm{eV}$ and $1.513 \mathrm{eV}$ may correspond to the 200 $\AA$ and $800 \AA$ well emissions but if this is so then their energies both shifted from the values prior to etching. By acquiring $\mathrm{CL}$ monochromatic images at the energies corresponding the supposed $200 \AA$ and $800 \AA$ well emissions from a region which included both etched and unetched areas, we detected a great decrease of CL intensity at $30 \mathrm{~K}$ in the etched areas. These changes in the emission line-shape and intensity from the etched areas suggest that the process of fabrication of the quantum dots induced damage on the directly etched surface, probably during the ion etching process.

Quantum-dots produced from the $500 \mathrm{~nm}$ diameter $50 \AA$ and $100 \AA$ quantum wells were examined by STEM-CL and the luminescence signal corresponding to the $50 \AA$ and $100 \AA$ wells was obtained from several individual dots. As may be deduced from figure 2 (curve (c)) no changes in the emission energies and FWHM were detected in comparison with the two-dimensional quantumwell emissions of curve (a). In the approximation of infinite potential barriers, the confinement energies for an electron or a hole in a rectangular-shape quantum-dot can be given as [12]

$$
\begin{gathered}
E=\frac{\pi^{2} \hbar^{2}}{2 m^{*}}\left(n_{1}^{2} / L_{x}^{2}+n_{2}^{2} / L_{y}^{2}+n_{3}^{2} / L_{z}^{2}\right) \\
n_{i}=1,2,3
\end{gathered}
$$

where $m^{*}$ is the effective mass of an electron or a hole; $E$ is the confinement energy measured respectively upward from the bottom of the conduction band or downward from the top of the 


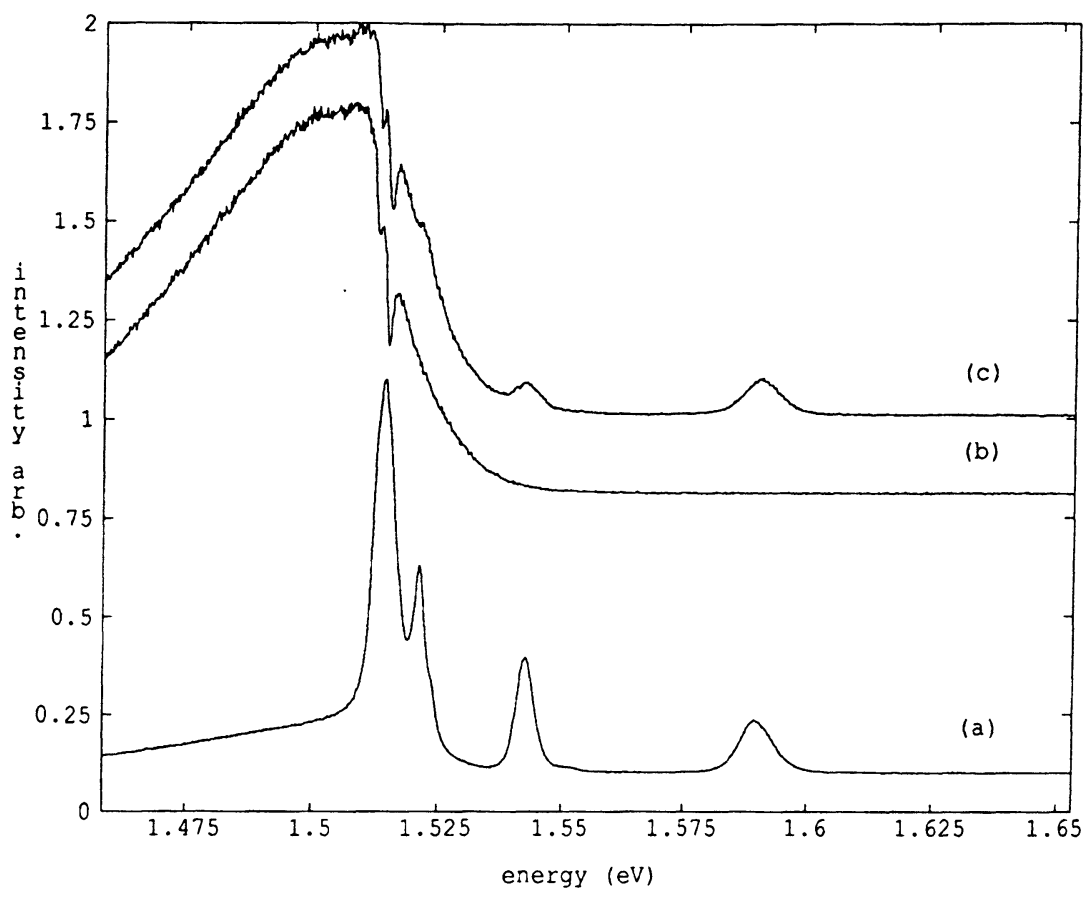

Fig. 2. - CL spectra: (a) from an unetched area representing the quantum-well emission; (b) from an etched area; (c) from an individual quantum-dot.

valence band and $L_{x}, L_{y}$ and $L_{z}$ are the vertical sizes and the lateral sizes of the quantum dot respectively. The ground state sub-band of the electrons in the conduction band or the holes in the valence band corresponds to $n_{i}=1$. The changes of the effective energy gap caused by lateral confinement of carriers for quantum-dots with diameters larger than $120 \mathrm{~nm}$ are less than $1 \mathrm{meV}$, so for $500 \mathrm{~nm}$ dots the energy shift would be unobservable in our experiments. Moreover, we observed a degradation of luminescence efficiency for the quantum-dots. There was a decrease by a factor of 0.5 for the $50 \AA$ wells and of 0.2 for the $100 \AA$ wells in comparison with the luminescence efficiency of the unetched structures. This degradation may be due to a high non-radiative recombination velocity at the sidewalls surface, but comparison with other research work [8], in which quantum-dots were fabricated from the same material as this work, the higher temperature applied in our experiment may also be responsible since temperature-enhanced diffusion enables more excitons to reach surface states. The difference between the degradation factors for the $50 \AA$ and $100 \AA$ wells may be explained by the lower carrier diffusion coefficient for the narrower quantum-wells which results from the carrier localisation effect [13]. This means that the narrower the QW, the smaller the number of carriers which could reach the sidewall surface, and hence the weaker the surface effect. The integrated CL intensities from the dots formed by the $100 \AA$ wells was also weak in comparison with that of the $50 \AA$ wells, as is shown by the CL monochromatic images in figure 3 . Image (a) in figure 3 was taken at an energy corresponding to the $50 \AA$ well emission while image (b) was taken from the $100 \AA$ well emission. The ratio of the dot-image intensity to the background was much higher for the dots formed by the $50 \AA$ wells than that for the $100 \AA$ wells. The bright patch at the left of the images was a processing fault and the dot marked by an arrow was missing in image (a) but not in image (b). 


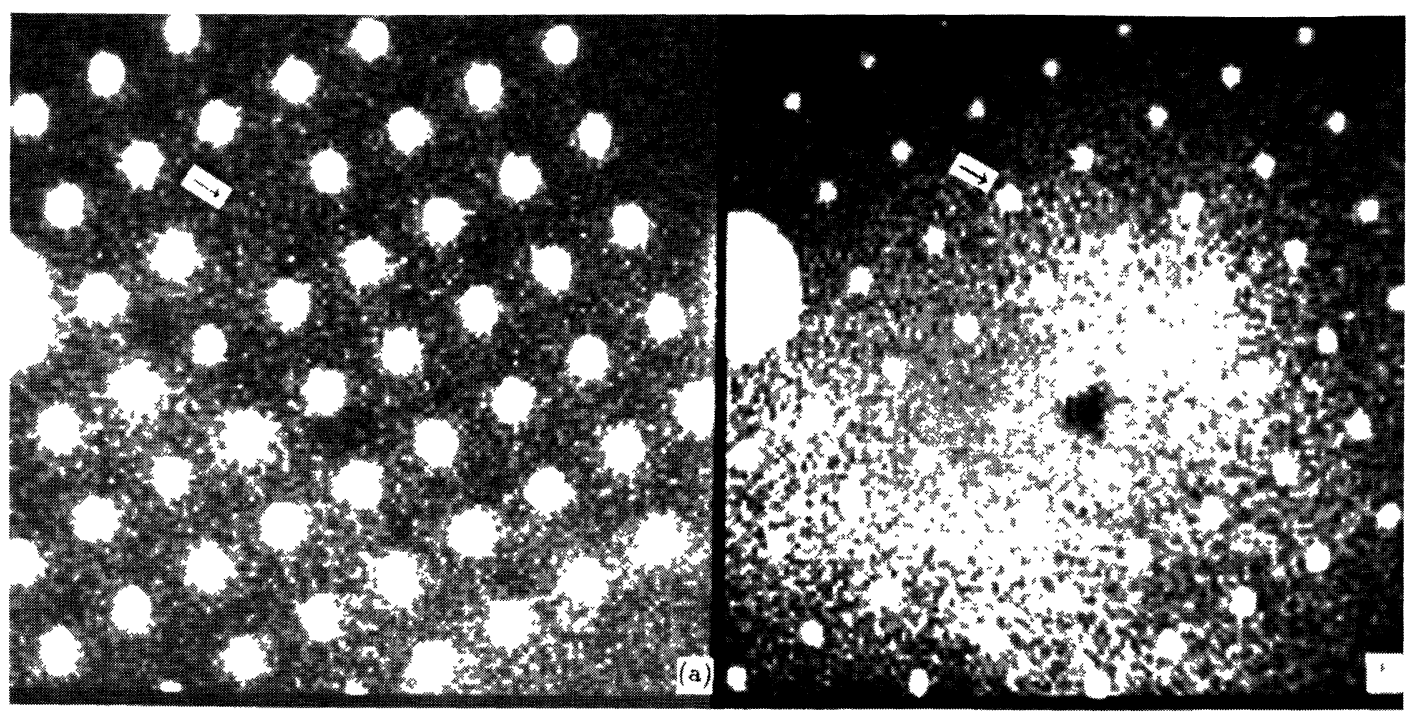

$10 \mu \mathrm{m}$

Fig. 3. - Energy-selected CL images acquired at: (a) $1.591 \mathrm{eV}$, the emission of the quantum-dots formed from $50 \AA$ quantum-well; (b) $1.543 \mathrm{eV}$, the emission of the quantum-dots formed from $100 \AA$ quantum well. The apparent difference in dot size in these images is not a genuine effect as can be established by line profiles through the intensity maps.

\section{Conclusion.}

Our studies of free-standing quantum dots, fabricated by reactive-ion-etching reveals that significant surface damage occured at the directly etched surfaces and indicates that high resolution STEM-CL is very useful for studying the performance of individual dots. A loss of luminescence from quantum dots in comparison with the original quantum-well structure represents a serious challenge to the observation of new phenomena induced by decreasing dimensionality of low dimensional structures.

\section{Acknowledgements.}

One of us (J.Wang) is grateful for the financial support of Chinese Academy of Science. Partial support for this project was provided by the Science and Engineering Research Council, U.K..

[2] Gibert J., Petroff P.M., Phys. Rev. B36 (1987) 3243.

[3] Petroff P.M., Inst. Phys. Conf. Ser. 87 (1987) 187. 
[4] Clausen E.M., Jr., Craighead H.G., Worlock J.M., Harbison J.P., Schiavone L.M., Florez L., VAN der GaAg B., Appl. Phys. Lett. 55 (1989) 1427.

[5] Demel T., Heitmann D., Grambow P., Ploog K., Appl. Phys. Lett. 53 (1988) 2176.

[6] KaSh K., Scherer A., Worlock J.M., Craighead H.G., TaMargo M.C., Appl. Phys. Lett. 49(1986) 1043.

[7] ARNot H., ANDREws S.R., BEAUMONT S.P., Proc. Microcirciut Engineering Conference (Vienna, Austria) 1988.

[8] Andrews S.R., ARnot H., ReEs P.K., KerR T.M., Beaumont S.P., J. Appl. Phys. 67 (1990) 3472.

[9] RoBerTs S., Inst. Phys. Conf. Ser. 60 (1981) 377.

[10] Myhajlenko S., BATSTONE J.L., Hutchinson H.T., SteEd J.W., J. Phys. C17 (1984) 6477.

[11] DAY J.C.C., Ph. D Thesis (1988).

[12] Wu W.Y., Schulman J.N., Hsu T.Y., Efron U., Appl. Phys. Lett. 51 (1987) 710.

[13] Hillmer H., Hansmann S., ForChel A., Morohashi M., LOPEZ E., MEIER H.P., PloOg K., Appl. Phys. Lett. 53 (1988) 1937. 